\title{
Genome-Wide Analysis of Almond Skp1 Family Members And Identification of Self-Incompatibility- Related PsdSSK1
}

\section{Zhenfan Yu}

Xinjiang Agricultural University

\section{Dongdong Zhang}

Xinjiang Agricultural University

Bin Zeng ( $\square$ zbxjau@163.com )

Xinjiang Agricultural University

Jiahui Yang

Xinjiang Agricultural University

\section{Wenwen Gao}

Xinjiang Agricultural University

\section{Yilian Tang}

Xinjiang Agricultural University

\section{Ayimaiti Abudukayoumu}

Xinjiang Agricultural University

\section{Xintong Ma}

Xinjiang Agricultural University

\section{Research Article}

Keywords: Almond, Skp1, genome-wide identification, expression analyses, PsdSSK1 clone, 30 Southern blot, Western blot

Posted Date: July 23rd, 2021

DOl: https://doi.org/10.21203/rs.3.rs-695099/v1

License: (c) (i) This work is licensed under a Creative Commons Attribution 4.0 International License. Read Full License 


\title{
Genome-wide analysis of almond $S k p 1$ family members and identification of self-incompatibility-related PsdSSK1
}

Zhenfan Yu+, Dongdong Zhang+, Bin Zeng*, Jiahui Yang, Wenwen Gao, Yilian Tang, Ayimaiti

Abudukayoumu, Xintong Ma

College of Forestry and Horticulture of Xinjiang Agricultural University, Urumqi, China

Yuzhenfan@126.com(Zhenfan Yu); a583787672@outlook.com(Dongdong Zhang); yangjiahui2021@126.com (Jiahui Yang); gwxjau313@163.com(Wenwen Gao); tangyilian21@163.com(Yilian Tang); abdukayyumjan@163.com(Ayimaiti Abudukayoumu); asherr0227@163.com(Xintong Ma)

*Corresponding to:Professor Bin Zeng, Collage of Forestry and Horticultural, Xinjiang Agricultural University, XinJiang Urumqi, 830052, P. R. China. E-mail: zbxjau@163.com, Tel: 0086-991-8787811, Fax: 0086-991-8762363

\begin{abstract}
S-phase kinase-associated protein 1 (Skp1) is a component of the SKP1-Cullin1-F-box (SCF) complex, which facilitates ubiquitin-mediated protein degradation in eukaryotes. SLF interacting Skp1-like 1 (SSK1), which is located outside the S-site, is a newly discovered type of self-incompatibility-related pollen-specific gene whose product interacts with pollen determinants. In this study, we identified 18 members of the Prunus dulcis Skp1(PsdSkp1) family that were unevenly distributed across six chromosomes of the almond genome. The phylogenetic tree revealed that the PsdSkp1 members could divided into four groups: I, II, III, and IV. The conserved motifs, conserved domains, and motif structures of the PsdSSK1 and PsdSkp1-12 proteins are similar. There were three pairs of fragment duplication genes and one pair of tandem repeat genes, and their functions were highly evolutionarily conserved. Transcriptome data showed that PsdSkp1 was expressed in almond flower tissues, that its expression was affected by freezing stress, and that its expression changed significantly in cross-pollination. Molecular experiments proved that PSdSSK1 and PsdSkp1-12 had the same protein sequence, and the genes were significantly highly expressed mainly in the pollen or throughout pollen cells, with subcellular localization in the cytoplasm. PsdSkp1-12 is an ssk1 gene in almond, and it may be involved in the SCF complex to regulate the almond pollen response via SI and cross-compatibility. In conclusion, our research lays a foundation for future functional research on PsdSkp1 members, especially for exploring the mechanism of almond SI.
\end{abstract}

Keywords: Almond; Skp1; genome-wide identification; expression analyses; PsdSSK1 clone; Southern blot; Western blot

\section{Introduction}

S-phase kinase-associated protein $1(S k p 1)$ is a small protein comprising approximately 160 amino acids and constitutes the core component connecting the CullinI and F-box proteins. As the core component of SCF-type E3 ubiquitin ligase, Skpl mediates the degradation of proteins by the $26 \mathrm{~S}$ proteosome and plays a key role in eukaryotic cell cycle progression, transcriptional regulation, signal transduction and many other cellular processes[1-3]. Only one Skpl gene has been found in protozoa, algae and fungi[4]. In contrast, plants have multiple Skp1 genes, which is the result of duplication events that led to functional differentiation[5,6]. In plants, $S k p 1$ genes play an important role in auxin, gibberellin, ethylene, and jasmonate signaling and in photoreactions[7-9], flower development and male meiosis[10,11]. In most cases, $S k p l$ functions by forming one of many SCF complexes together with Cullin1, Rbxl and F-box proteins. Arabidopsis 
thaliana Skp1 (ASK) proteins play an important role in the development of flowers and the sterility of male gametophytes[12-14]. Solanum lycopersicum Skp1 (SSK) proteins are involved in the development of tomato fruits[15], and Triticum aestivum Skp1 (TSK) proteins regulate male meiosis in wheat[16]. Overexpression of Paeonia suffruticosa Skp1 (PSK1) was shown to increase the expression of the flowering-related genes CO, LEAFY and FT and promoted flower formation and early flowering. The expression of PSK1 gradually increases with the development of flower buds and the flowering process[17]. Transgenic Arabidopsis plants overexpressing PSK1 exhibit increased salt tolerance due to increased accumulation of proline and soluble sugars[17]. This effect was similar to that of $A S K 1$, which interacts with B-function proteins (B subfamily MADS-box gene products) and cooperates with $U F O$ and $L E A F Y$ proteins in flower development and floral organ identity[13]. In Arabidopsis flowers expressing mutant ASK1 genes, increased expression of two proteins was detected: one is a class I heat-shock protein induced in response to stress and provides protection against oxidative damage to cells; the other is an oxygen-evolving complex-related protein. These findings show that mutations in ASK1 may directly or indirectly cause oxidative stress in floral organs[18]. In upland cotton (Gossypium hirsutum), strong expression of GhSKPl in the radicle and stamen that comprise dividing cells showed that GhSKP1 may be involved in the growth and flower development. ABA had a slight inhibitory effect on the expression of GhSKPl in cotton leaves[19]. Rice OSK1 and tomato SSK1 and SSK2 are also involved in leaf senescence[20].

A newly discovered Skpl-like protein was discovered in Antirrhinum hispanicum. SLF-interacting Skp1-like 1 (SSK1), located outside the S-site, is a new type of self-incompatibility-related pollen-specific gene that interacts with pollen determinants[21]. Researchers analyzing the composition of the snapdragon (Antirrhinum hispanicum) SCF complex found that AhSSK1 can interact with AhSLF-S2 and CUL-like proteins and that AhSSK1 can form an SCF complex composed of SSK1, CUL1, SLF and Rbx1[21]. In 2010, Zhao Lan et al. [22] also identified and cloned the AhSSK1 homologous gene from Petunia hybrida, PhSSK1. The two genes were $48.3 \%$ homologous. The same in vitro biochemical experiment proved its interaction with the pollen self-incompatibility (SI) determinants PhSLF, Cullin1 and S-RNase. These findings show that SSK1 acts as a linker within the SCF complex, is responsible for connecting the F-box protein, and indicates that SSK1 is involved in the SI signal response. In addition, transgenic RNA interference (RNAi) experiments showed that downregulation of PhSSK1 affected the pollen affinity response, 
and partial segregation was also detected in a genetic analysis of transgenic offspring. In plantain and Solanaceae species, it has been found that there is a pollen-specific SSK1 that interacts together with SLF in the SLF-mediated SCF complex and that this SSK1 is required to degrade S-RNase[22]. In addition, in 2012, Xu and Li [23] identified and cloned two SSK1 genes from Pyrus bretschneideri (Rosaceae), PbSSK1 and PbSSK2. Homologs of the Skpl-like gene have also been cloned from apple, pear and sweet cherry; and the products of all these genes can bind to their corresponding SLF and Cullinl proteins[24,25]. Recently, Zeng et al. [26] cloned SCF complex-related genes from Prunus tenella (PetSSK1 and PetSBP1), and using yeast two-hybrid and pulldown experiments, the researchers found that PetSBP1 mainly connected the Cullin1 protein and F-box protein together in wild almond, leading to the formation of a functional SCF complex.

Almond (Rosaceae: Prunus dulcis) is an economically important crop tree species that exhibits gametophytic self-incompatibility (GSI)[27]. SI is the most important mechanism controlling plant self-fertilization[28] and has been reported in more than half of all flowering plants and most other Rosaceae species[29,30]. SI refers to the ability of the pistil of a plant to identify and inhibit the germination and growth of its own pollen, in which the E3 ubiquitin ligase $\mathrm{SCF}^{\mathrm{SFB}}$ (SLF) complex plays a decisive role. The SCF complex has a spherical structure and consists of four main components: Skp1, Cullin1, Rbx1, and F-box proteins. The mechanism of S nuclease-based GSI involves tagging a target protein for entry into the ubiquitin proteasome pathway (UPP), thereby causing it to be broken down by the $26 \mathrm{~S}$ proteasome, which ultimately drives SI[31].

To date, Skpl family genes and SSK1 genes have not been reported in almond. To explore the characteristics of almond Skpl family members, we used almond genome-wide data to screen Skpl family members. The physicochemical properties, subcellular localization, phylogenetic relationships, conserved protein motifs, conserved domains, gene structure, chromosomal gene distribution, collinear genes and cis-elements were analyzed. Transcriptome data from different tissues of almond, anthers and ovaries under freezing stress, and self- and out-crossed pollen were obtained to calculate the fragments per kilobase per million mapped read (FPKM) values and analyze expression patterns. To determine whether members of the $S k p 1$ family in almond have SSK1 function, we cloned the almond PsdSSK1 gene and assessed the in vitro expression of almond pollen by fluorescence quantification and Southern blot and Western blot analyses. This study provides theoretical data for the functional role of $S k p 1$ family genes in almond, provides a 
111 theoretical basis and data for determining whether the SSK1 gene is involved in the SI 112 pathway of almond, and is useful for investigating the SI of almond. The mechanism of

113 action responsible is highly important.

\section{Results}

\subsection{Genome-wide identification of the $S k p 1$ genes in almond}

Eighteen Skp1 genes were identified and screened in almond. These genes were renamed PsdSkp1-1 PsdSkp1-17 according to their chromosome and location, and two of them were homologous genes, which were renamed PsdSkp1-8a and PsdSkp1-8b. The physicochemical properties of the proteins showed that the number of amino acids ranged from 93 (PsdSkp1-2) to 367 (PsdSkp1-8b) amino acids, and the molecular weight ranged from 10791.13 (PsdSkp1-2) to 42582.44 (PsdSkp1-8b) Da. The isoelectric point ranged from 4.41 to 5.69 , and the hydrophilic and hydrophobic values were all less than 0 , indicating that the proteins are hydrophilic. Subcellular mapping showed that PsdSkp1-8b was located in the mitochondrion, PsdSkp1-5 was located in the nucleus, PsdSkp1-9 and PsdSkp1-16 were located in the chloroplast, and the other 14 proteins were located in the cytoplasm.

128 Table 1 Detailed information on the PsdSkp1 gene family members in almond

\begin{tabular}{|c|c|c|c|c|c|c|c|c|c|}
\hline Gene name & Gene ID & Chromosome & Start & End & Number of & Molecular & Isoelectric & GRAVY & Subcellular \\
\hline & & & & & amino acids & weight/Da & point & & localization \\
\hline PsdSkp1-1 & Prudul26A001581C1 & Pd01 & 29822524 & 29823973 & 156 & 17767.94 & 4.53 & -0.597 & Cytoplasm \\
\hline PsdSkp1-2 & Prudul26A010302C1 & Pd02 & 7447046 & 7447384 & 93 & 10791.13 & 4.42 & -0.674 & Mitochondrion \\
\hline PsdSkp1-3 & Prudul26A004902C1 & Pd02 & 11802849 & 11803382 & 177 & 20194.72 & 4.61 & -0.779 & Cytoplasm \\
\hline PsdSkp1-4 & Prudul26A003178C1 & $\mathrm{Pd} 02$ & 11871398 & 11871868 & 156 & 18027.36 & 4.46 & -0.556 & Cytoplasm \\
\hline PsdSkp1-5 & Prudul26A017928C1 & $\mathrm{Pd} 02$ & 11878821 & 11879288 & 155 & 17776.12 & 4.57 & -0.483 & Nucleus \\
\hline
\end{tabular}




\begin{tabular}{|c|c|c|c|c|c|c|c|c|c|}
\hline PsdSkp1-6 & Prudul26A022605C1 & $\mathrm{Pd} 02$ & 11895200 & 11895670 & 156 & 17882.14 & 4.41 & -0.503 & Cytoplasm \\
\hline PsdSkp1-7 & Prudul26A000183C1 & $\mathrm{Pd} 02$ & 12798666 & 12799205 & 179 & 20236.17 & 4.97 & -0.375 & Cytoplasm \\
\hline PsdSkp1-8a & Prudul26A002877C1 & $\mathrm{Pd} 02$ & 17496362 & 17500871 & 340 & 39478.61 & 5.33 & -0.861 & Cytoplasm \\
\hline PsdSkp1-8b & Prudul26A002877C2 & Pd02 & 17496362 & 17502012 & 367 & 42582.44 & 5.69 & -0.807 & Cytoplasm \\
\hline PsdSkp1-9 & Prudul26A026206C1 & $\mathrm{Pd} 02$ & 19991933 & 19992358 & 141 & 16037.53 & 5.07 & -0.035 & Chloroplast \\
\hline PsdSkp1-10 & Prudul26A016207C1 & Pd02 & 24563273 & 24568462 & 356 & 40766.03 & 5.45 & -0.795 & Cytoplasm \\
\hline PsdSkp1-11 & Prudul26A026600C1 & Pd04 & 3293555 & 3294014 & 131 & 15261.61 & 5.16 & -0.463 & Cytoplasm \\
\hline PsdSkp1-12 & Prudul26A027568C1 & Pd04 & 7760346 & 7761334 & 177 & 20538.04 & 4.52 & -0.567 & Cytoplasm \\
\hline PsdSkp1-13 & Prudul26A018869C1 & Pd06 & 4839281 & 4841071 & 98 & 11291.95 & 5.12 & -0.148 & Cytoplasm \\
\hline PsdSkp1-14 & Prudul26A031483C1 & Pd07 & 3083813 & 3084244 & 118 & 13706.56 & 4.72 & -0.469 & Cytoplasm \\
\hline PsdSkp1-15 & Prudul26A008750C1 & Pd07 & 16186460 & 16188084 & 156 & 17685.92 & 4.57 & -0.535 & Cytoplasm \\
\hline PsdSkp1-16 & Prudul26A016175C1 & $\mathrm{Pd} 07$ & 16194299 & 16195427 & 157 & 17936.47 & 4.77 & -0.417 & Chloroplast \\
\hline PsdSkp1-17 & Prudul26A019975C1 & Pd08 & 14876840 & 14878185 & 157 & 18049.32 & 4.54 & -0.424 & Cytoplasm \\
\hline
\end{tabular}

131 members of 10 species was divided into four groups on the basis of the results of clustering

132 branching: groups I, II, III, and IV (Figure 1). There was only one PsdSkp1-13 in group I;

133 in group II, PsdSkp1-8a, PsdSkp1-8b, and PsdSkp1-10 were closely related and clustered

134 together with $A S K 20$ and $A S K 21$, suggesting they may be homologous proteins with similar

135 functions. PsdSkp1-9, PsdSkp1-11, and PsdSkp1-12 as well as SSK1 genes from all the

136 species were in group III. Among them, PsdSkp1-12 is closely related to FaSSK1, Pu $x$ 137 PcSSK1, MdSSK1, PaSSK1, PtSSK1, and PpSSK1 of the Rosaceae family; these are 138 homologous proteins and may be encoded by SSK1 genes. Group IV contained 19 ASK 
members and 11 PsdSkp1 members. Among them, PsdSkp1-1, PsdSkp1-15, and PsdSkp1-16 were closely related and clustered together with ASK2. The remaining PsdSkp1 members clustered relatively closely together with $A S K 3$ and $A S K 4$, while all the other $A S K$ members were clustered together.

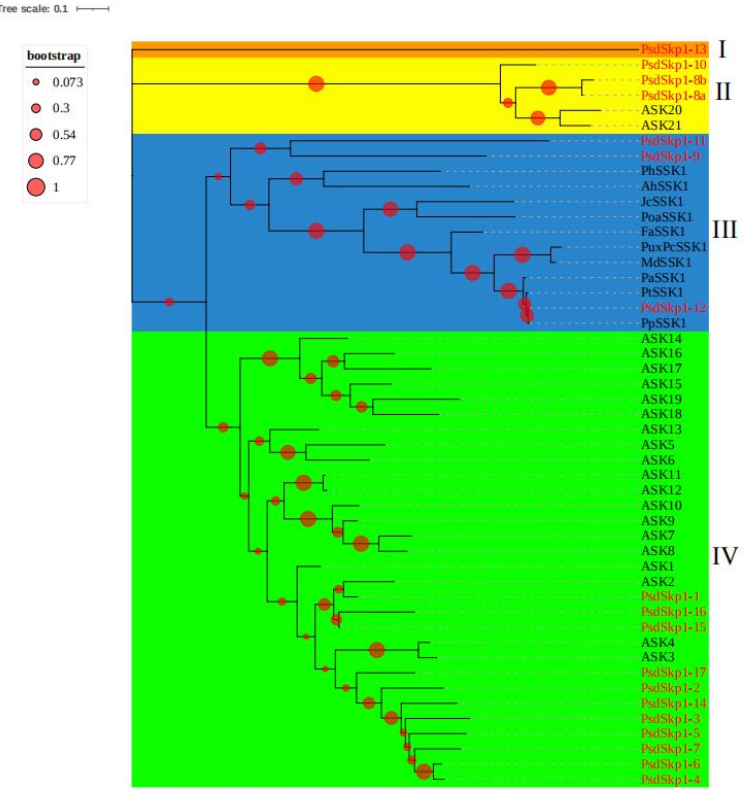

Figure 1 Phylogenetic tree of Skpl family members and SSK1 from almond and Arabidopsis thaliana. The evolutionary history was inferred using the maximum likelihood method and a JTT matrix-based model. This analysis involved 49 amino acid sequences. The blocks of different colors represent different group members. ASK (Arabidopsis thaliana:Skp1); AhSSK1 (Antirrhinum hispanicum); PhSSK1 (Petunia hybrida); JcSSK1 (Jatropha curcas); PoaSSK1 (Populus alba); FaSSK1 (Fragaria ananassa); PuxPcSSK1 (Pyrus ussuriensis x Pyrus communis); MdSSK1 (Malus domestica); PaSSK1 (Prunus avium); PtSSK1 (Prunus tenella); PpSSK1 (Prunus persica).

\subsection{Motifs, domains and gene structure of PsdSkp1 gene members}

The conserved motifs of PsdSkp1 family members were annotated to 20 conserved motifs. Motif 1 was considered a Skp1 type, and motif 2, motif 3, and motif 11 were of the Skp1_POZ type (Figure 2E). Cluster analysis via a phylogenetic tree showed that in PsdSkp1-13 in group I, motif 11, motif 12, and motif 20 were conserved. In group II, PsdSkp1-11 contained motif 1, while PsdSkp1-9 and PsdSkp1-12 contained motif 1 and motif 11. In group III, PsdSkp1-8a, PsdSkp1-8b, and PsdSkp1-10 contained motif 1, motif 2, 
159

160

161

162

163

164

165

166

167

168

169

170

171

172

173

174

motif 4 , motif 6 , and motif 7 , the distributions of which were highly conserved. In group IV, 11 PsdSkpl proteins contained motif 1 , motif 5 and motif 8 , and 8 PsdSkpl proteins contained motif 2 (Figure 2B).

The conserved domains of the PsdSkp1 family members showed that, except in the case of PsdSkp1-13 and PsdSkp1-2, there were no Skp1_POZ types; the other 16 PsdSkp1 proteins contained a Skp1_POZ domain. Although PsdSkp1-13 has only one Skp1 superfamily domain, the other 15 PsdSkpl proteins contained 2-3 conserved domains, which are of the Skp1_POZ types, Skp1 and Skp1 superfamilies (Figure 2C). The results were similar to those obtained with respect to the conserved motifs.

Cluster analysis of gene structure and the phylogenetic tree revealed that, in group I, PsdSkp1-13 had three exons and two introns. In group II, PsdSkp1-9, PsdSkp1-11 and PsdSkp1-12 had one exon and 0 introns. In group III, PsdSkp1-8a and PsdSkp1-8b had 8 exons and 7 introns, whereas PsdSkp1-10 had 9 exons and 8 introns. In group IV, 5 PsdSkp1 members had 1 exon and 0 introns, and 6 PsdSkp1 members had 2 exons and 1 intron (Figure 2D).

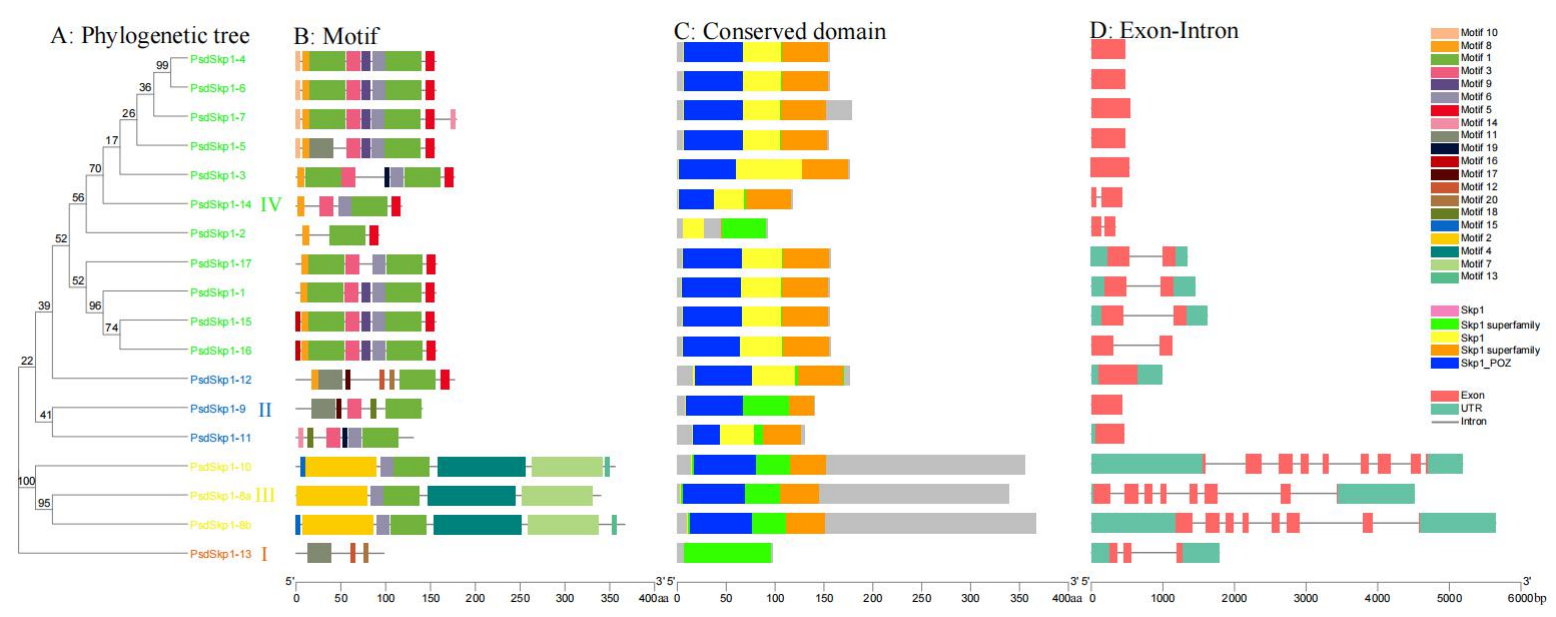



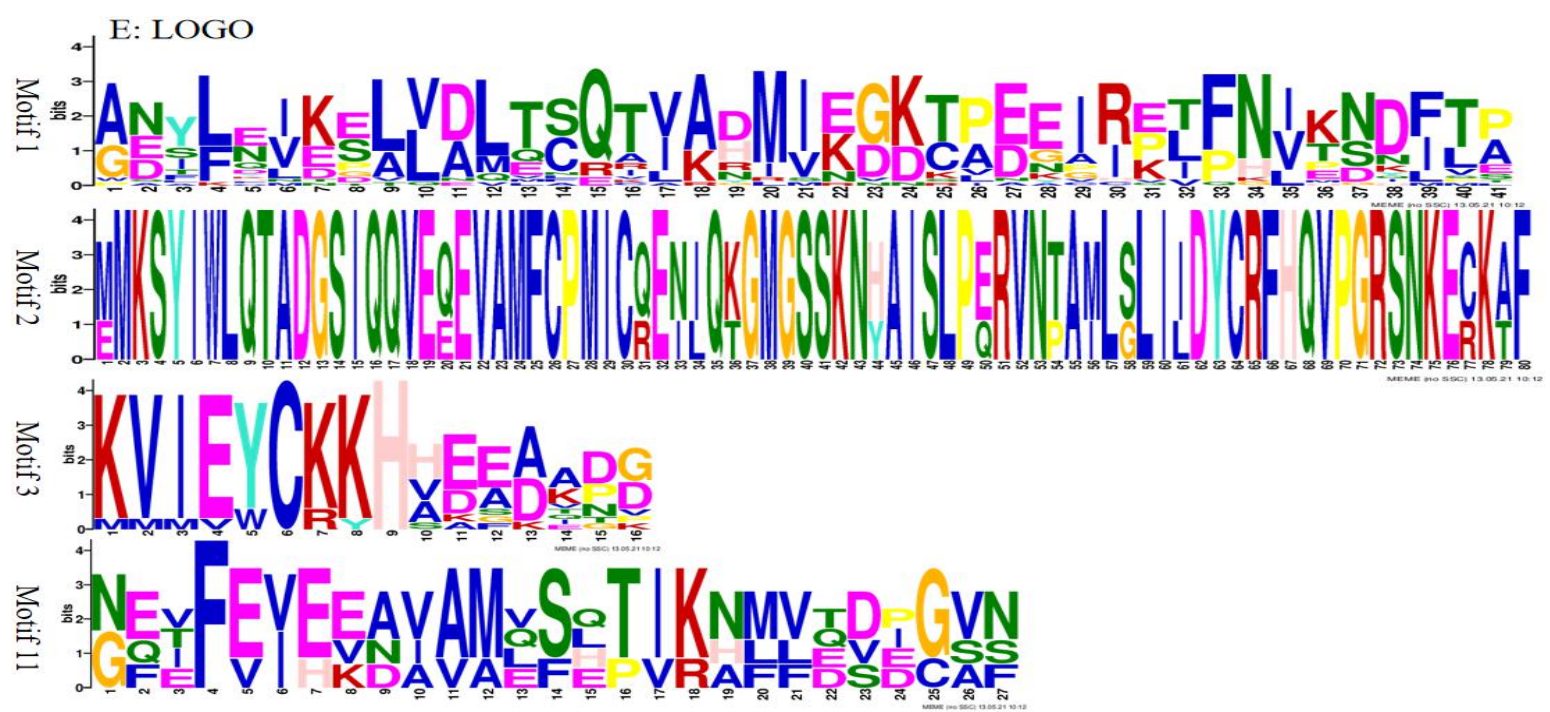

Figure 2 Phylogenetic clustering of PsdSkpl gene members. (A) Phylogenetic tree of

PsdSkp1 family members divided into four groups: I, II, III, and IV. (B) Conserved protein motifs. (C) Conserved protein domains. (D) Gene structure: exon-intron. (E) Motif 1, motif 2, motif 3 and motif 11 conservation according to LOGO.

\subsection{Chromosomal distribution and analysis of collinear genes of PsdSkp1 gene members}

PsdSkp1 gene members were distributed across six chromosomes: Pd01, Pd02, Pd04, Pd06, Pd07, and Pd08 (Figure 3A). One PsdSkp1 gene was found on Pd01, Pd06, and Pd08 each; two PsdSkp1 genes were distributed on chromosome Pd04; three PsdSkp1 genes were distributed on chromosome Pd07; and 10 PsdSkpl genes were distributed on chromosome Pd02, which may have similar functions.

Chromosomal collinearity analysis revealed that 18 PsdSkp1 members had three pairs of segmental duplicated genes, PsdSkp1-1/PsdSkp1-15, PsdSkp1-1/PsdSkp1-17, and PsdSkp1-15/PsdSkp1-17, which were distributed on chromosomes Pd01, Pd07, and Pd08. At the same time, a pair of tandem repeat genes, PsdSkp1-8a/PsdSkp1-8b, were found on chromosome Pd02. Almond had 7, 18, 21, and 11 pairs of genes that were collinear with those in Arabidopsis thaliana, Malus domestica, Prunus persica, and Prunus mume, respectively (Figure 3C). In addition, the $\mathrm{Ka} / \mathrm{Ks}$ values of three pairs of duplicated fragments, one pair of tandem repeat genes, and one pair of collinear genes among the species were less than 1 , indicating that almond was affected mainly by purification and that the gene functions were highly conserved (Supplement Table 1). 
196

197

198

199

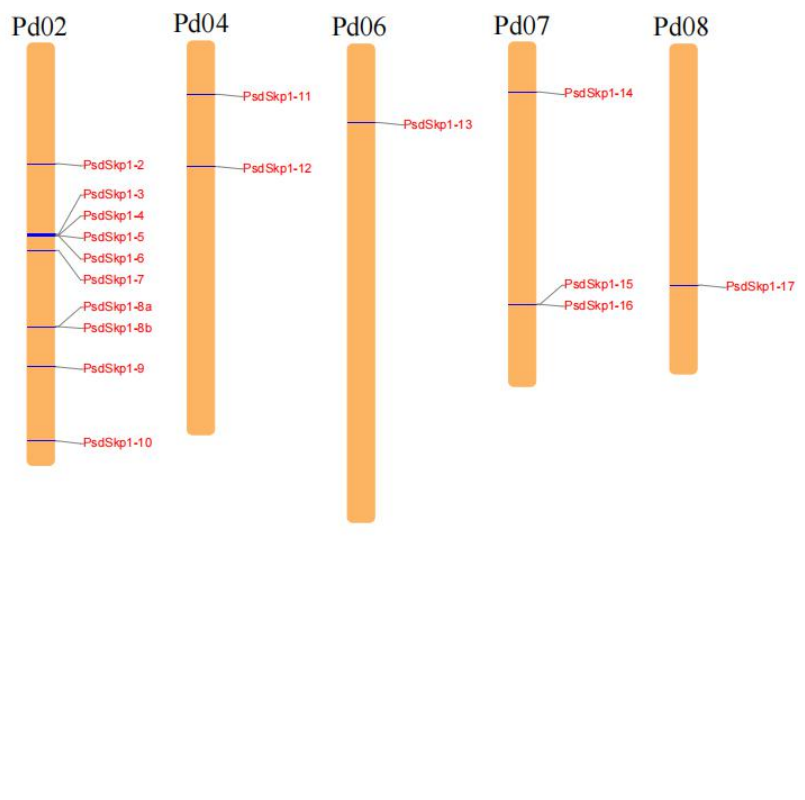

Figure 3A Chromosomal distribution of PsdSkpl genes in almond. The scale on the left shows the chromosome length information, and the chromosome names are listed at the top.

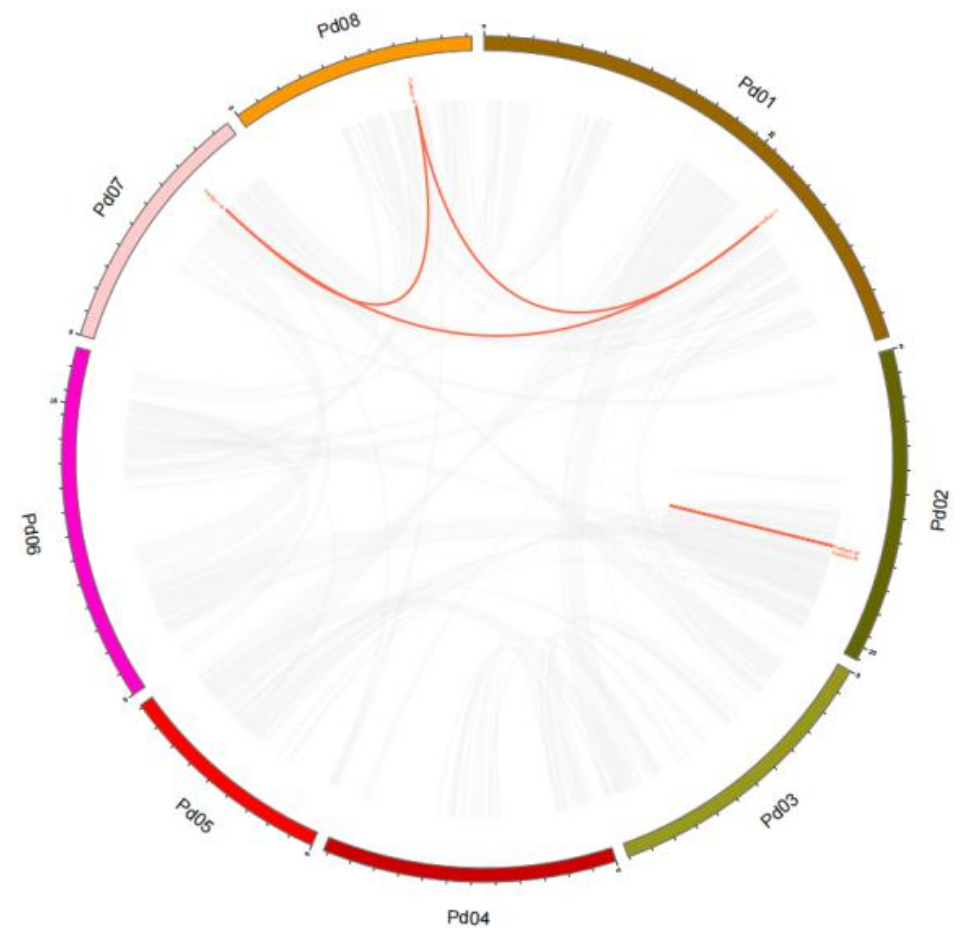

Figure 3B Distribution of duplicated gene pairs in the almond genome. Both genes of the same duplicated gene pair are labeled in red. 

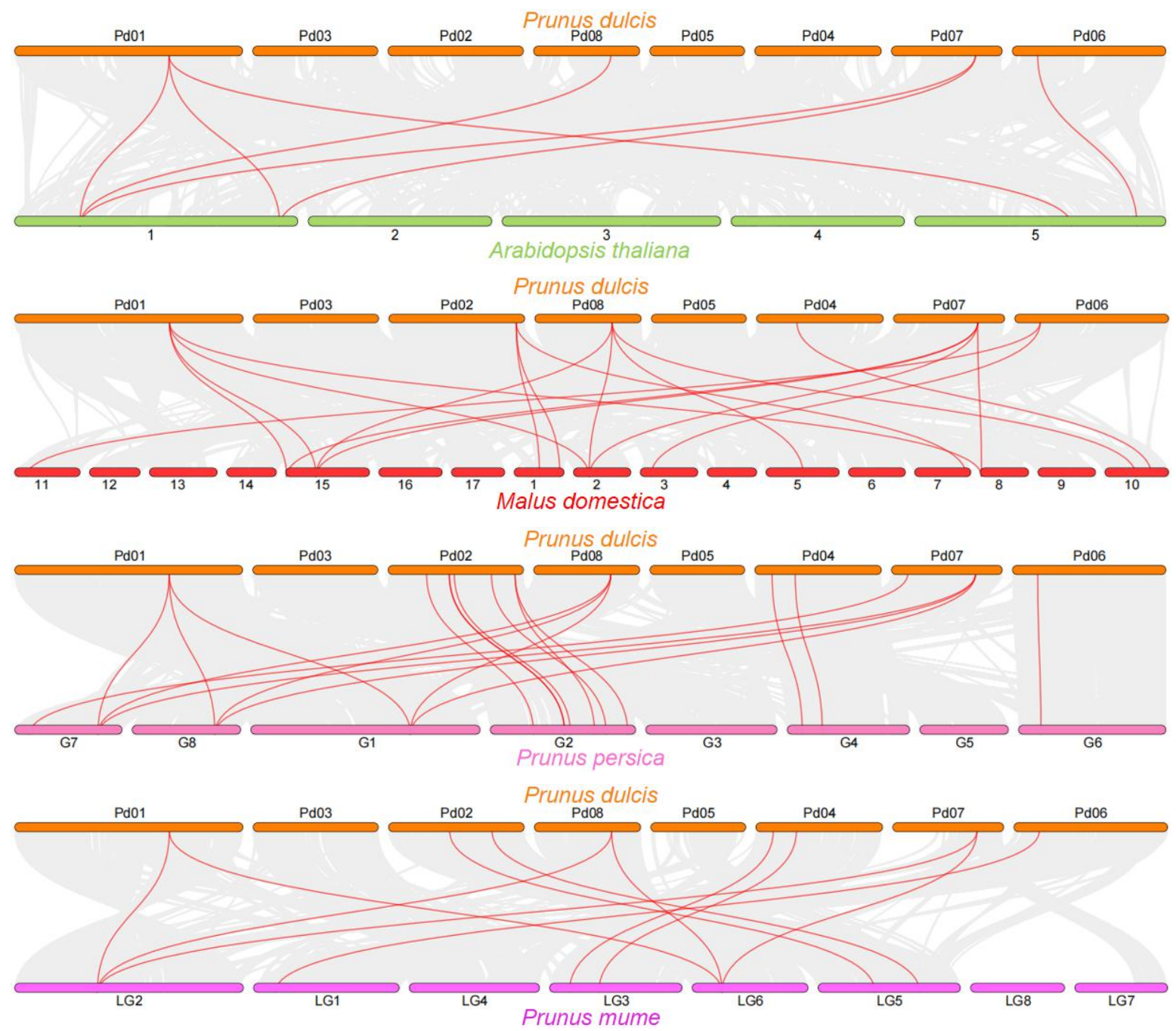

Figure 3C Syntenic relations of $S k p 1$ gene members among almond and four representative plant species. The gray lines in the background indicate collinear blocks within the almond genome and other plant genomes, and the red lines highlight syntenic Skp1 gene pairs.

\subsection{Upstream cis-regulatory elements}

The 2000 bp promoter region upstream of 18 PsdSkp1 family members in almond was annotated to a large number of core elements (TATA-boxes and CAAT-boxes) and light-responsive elements. In addition, there were various components associated with growth and development, hormone responses, and stress resistance (Figure 4). The GCN4

215 motif, O2 site, circadian element, CAT-box, Unnamed_1 element, Ry element, MSA-like

216 element, and MBSI element were found to be related to growth and development. Additionally, there were six types of elements related to the hormone response, TGACG 

types of stress-related components ARE MBSs, AREs, LTRs, and TC-rich repeats.

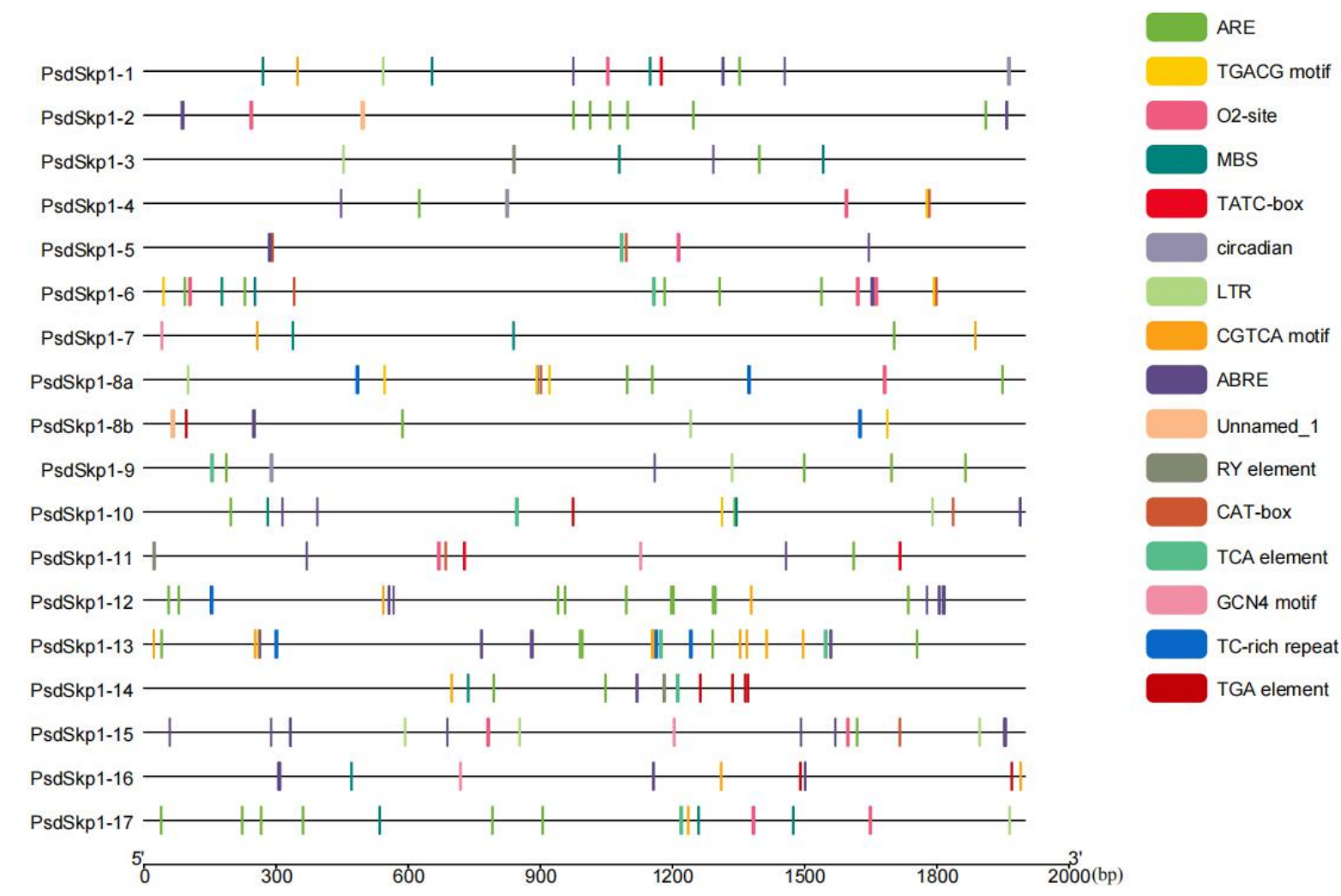

Figure 4 Cis-elements in promoter region of PsdSkp1 members. The colored boxes indicate the types of cis-elements.

\subsection{Expression analysis of PsdSkp1 gene members}

The PsdSkp1-2, PsdSkp1-4, PsdSkp1-5, PsdSkp1-6, PsdSkp1-9, and PsdSkp1-14 genes were not expressed in the leaf, flower bud, pistil, or fruit tissues (Figure 5A). In the leaves, all the PsdSkp1 family members were downregulated. In the flower buds, the PsdSkp1-8a, PsdSkp1-10, PsdSkp1-13, PsdSkp1-15, and PsdSkp1-17 genes were significantly highly expressed, the PsdSkp1-1 and PsdSkp1-16 genes were expressed at low levels, and other genes were downregulated. In the pistils, the expression of PsdSkp1-7, PsdSkp1-8a, PsdSkp1-11, PsdSkp1-12, and PsdSkp1-16 was significantly higher than that of PsdSkp1-1, PsdSkp1-10, and PsdSkp1-13. In the fruitlets, the PsdSkp1-1, PsdSkp1-3, PsdSkp1-8b, and PsdSkp1-17 genes were highly expressed, and the expression of other genes decreased.

233 These results indicate that PsdSkpl family members exhibit obvious tissue-specific 234 expression characteristics. Under control and freezing-stress conditions, five genes, 
PsdSkp1-2, PsdSkp1-5, PsdSkp1-9, PsdSkp1-11, and PsdSkp1-14, were not expressed (Figure 5B). In control anthers, PsdSkp1-7 and PsdSkp1-12 were significantly highly expressed, while the other genes were downregulated. In the anthers under freezing stress, the expression of PsdSkp1-6 increased sharply, and PsdSkp1-8b expression increased, but the expression was low. The expression of PsdSkp1-12 decreased and was weak; the expression of other genes decreased. In the control ovaries, the PsdSkp1-1, PsdSkp1-3, PsdSkp1-4, PsdSkp1-8a, PsdSkp1-8b, and PsdSkp1-10 genes were significantly highly expressed, while the other genes were downregulated. In anthers under freezing stress, the downregulated expression of PsdSkp1-1, PsdSkp1-3, PsdSkp1-4, PsdSkp1-8a, PsdSkp1-8b, and PsdSkp1-10 was inhibited, while the upregulated expression of PsdSkp1-13, PsdSkp1-15, PsdSkp1-16 and PsdSkp1-17 was significant. Taken together, these results indicate that the expression levels of some members of the PsdSkpl family change when anthers and ovaries are subjected to freezing stress to cope with the resulting adverse effects. An expression heat map of the four types of pistil pollination (A, AXA, I, and IxA) showed (Figure 5C) that the expression of six genes, PsdSkp1-2, PsdSkp1-5, PsdSkp1-7, PsdSkp1-9, PsdSkp1-11 and PsdSkp1-14, was inhibited. In A, six genes, PsdSkp1-1, PsdSkp1-3, PsdSkp1-4, PsdSkp1-6, PsdSkp1-8b and PsdSkp1-17, were significantly highly expressed; three genes, PsdSkp1-10, PsdSkp1-15 and PsdSkp1-16, were expressed at low levels; and the other genes were downregulated. In I, only two genes, PsdSkp1-16 and PsdSkp1-17, were expressed at low levels, while the other genes were downregulated. In AXA, the PsdSkp1-1, PsdSkp1-3 and PsdSkp1-16 genes were expressed, PsdSkp1-8a and PsdSkp1-10 expression was low, and the expression of the other genes was downregulated. Compared with that in AXA, the expression of the PsdSkp1-8A, PsdSkp1-12, PsdSkp1-13, and PsdSkp1-15 genes in IXA significantly increased. 


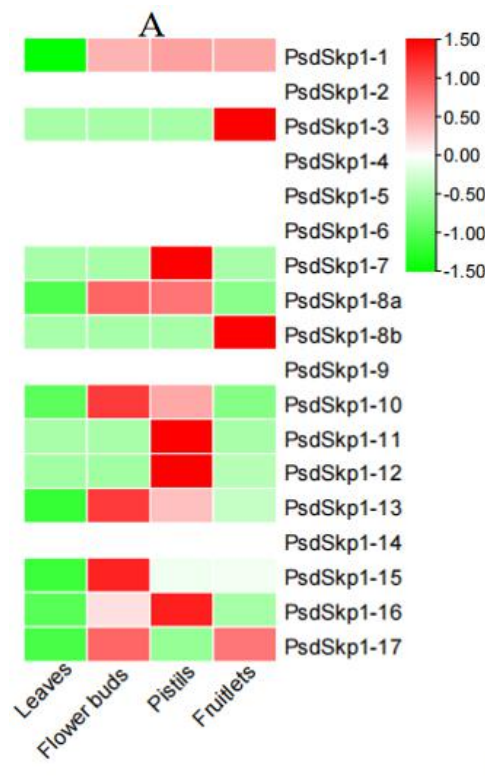

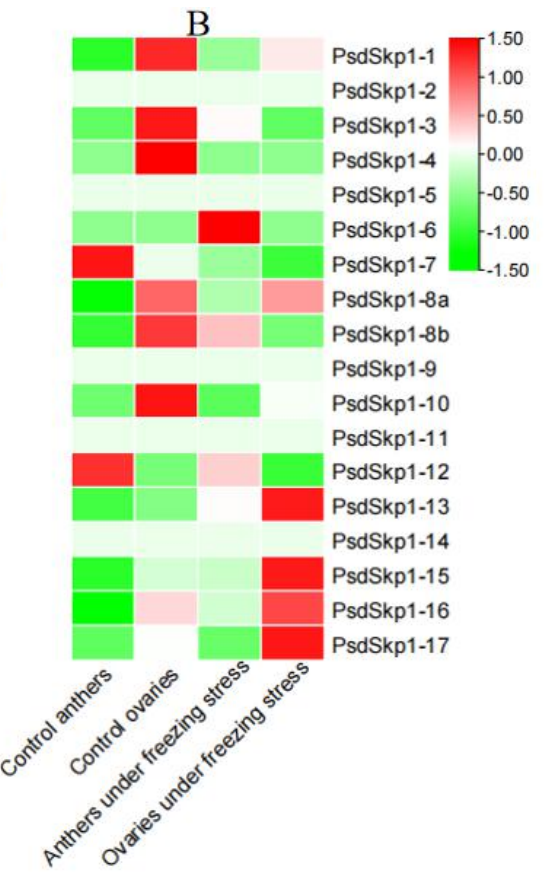

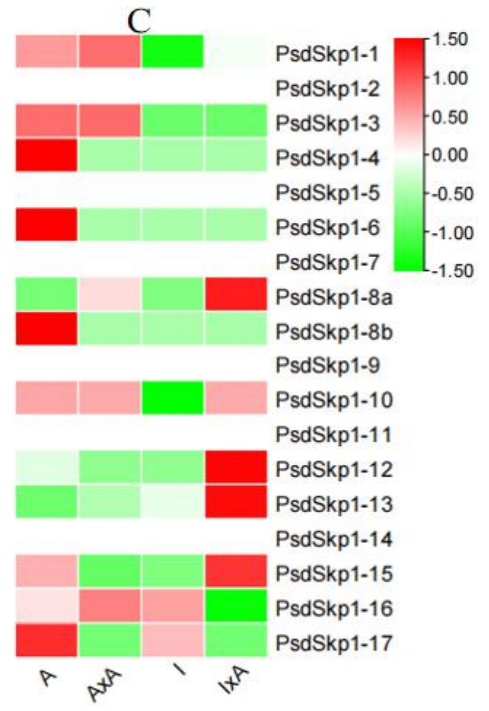

Figure 5 Expression of PsdSkp1 family members. (A) Expression levels in the leaves, flower buds, pistils, and fruitlets. (B) Expression levels in control anthers, control ovaries, anthers under freezing stress, and ovaries under freezing stress. (C) A: Unpollinated A2-198 pistils, I: Unpollinated ITAP-1 pistils, AxA: Self-pollinated A2-198 pistils, IxA: ITAP-1 pistils pollinated with A2-198. The row normalization method was used to construct the heat map.

\subsection{PsdSSK1 cloning}

In accordance with the designed primers, agarose gel electrophoresis was used to detect RNA samples from cultivated almond. PCR was used to clone and amplify the full-length (585 bp) sequence (Figure 6A) of the PsdSSK1 gene-coding region, which was consistent with the corresponding PsdSkp1-12 protein sequence. Via analysis of its open reading frame (ORF), the PsdSSK1 gene, whose conserved domains included a 62 amino acid Skp1_POZ domain and a 47 amino acid Skp1 domain (Figure 6B), was 534 bp in length, encoding 178 amino acids (Figure 6C). Multiple alignments of the PsdSSK1 protein sequence with the those of 8 other species showed that all of them contained Skp1_POZ and Skp1 domains (Figure 6D), indicating that our results concerning PsdSSK1 were extremely reliable. The physicochemical properties revealed that the PsdSSK1 protein was hydrophilic, with a molecular weight of $20538.04 \mathrm{Da}$, an isoelectric point of 4.52 , a 
molecular formula of $\mathrm{C}_{910} \mathrm{H}_{1421} \mathrm{~N}_{233} \mathrm{O}_{295} \mathrm{~S}_{6}$, a stability index of 41.48, an aliphatic index of 83.11, and a grand average of hydropathy of -0.567 .

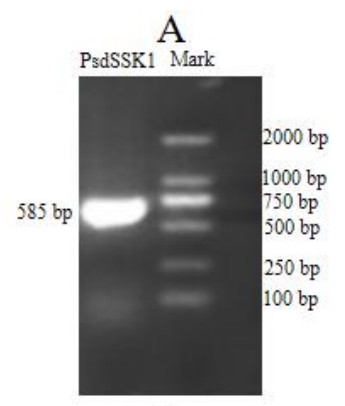

$\mathrm{C}$

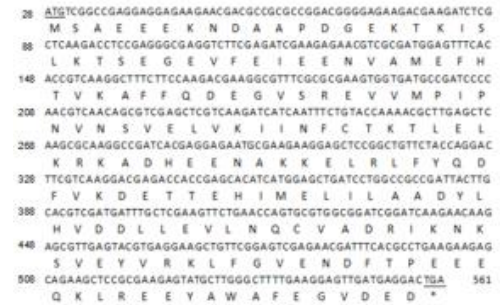

B

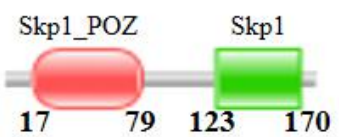

$\mathrm{D}$

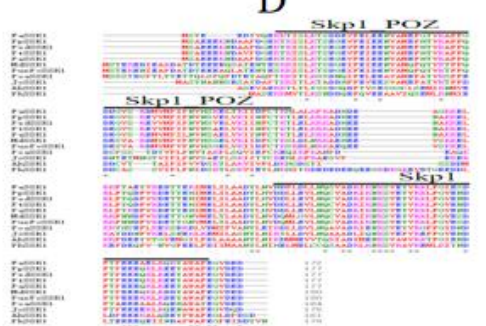

Figure 6 (A) PCR amplification of the PsdSSK1 gene. (B) PsdSSK1 conserved domains. (C) PsdSSK1 cDNA sequence and amino acid sequence. (D) Multiple alignment of sequences of SSK1 proteins from multiple species.

2.8 Expression of the PsdSSK1 Gene in Different Tissues of Cultivated Almond and Southern and Western blot Detection

Almond $\beta$-actin was selected as the internal reference gene. The amplification curve and dissolution curve of the actin gene and the PsdSSK1 gene showed that the dissolution curves of each showed a single peak, indicating that the specificity of the primers was strong. During the PCR amplification process, no nonspecific amplification, such as that reflecting the presence of primer dimers, occurred, which meant the results were suitable for qPCR analysis. The tissue samples were standardized and analyzed using the $2^{-\triangle \Delta \mathrm{CT}}$ method. In the figures, error bars represent the means \pm standard deviations. The expression level of the PsdSSK1 gene was highest in pollen tissue, while the expression levels in other tissues (petals, sepals, styles and leaves) were relatively low (Figure 7B; Figure 7C).

Southern blotting of almond cultivated in Xinjiang was performed to determine the copy numbers of DNA. The Southern blot results of the PsdSSK1 gene revealed the 
following (Figure 7D): PsdSSK1 gene is present as single copy in the leaves, petals, style, sepals and pollen.

Analysis of the PsdSSK1 protein expression in the five tissues of almond cultivated in Xinjiang according to Western blot analysis revealed that (Figure 7E) the PsdSSK1 protein was expressed in the petals, styles, and pollen tissues, and the expression of PsdSSK1 in the pollen was significantly higher than that in the other tissues.

A

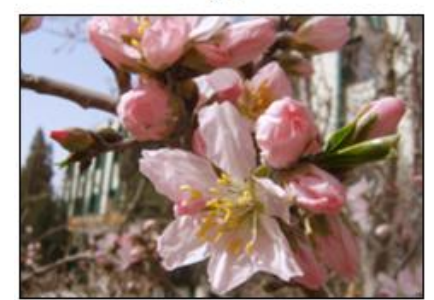

$\mathrm{C}$

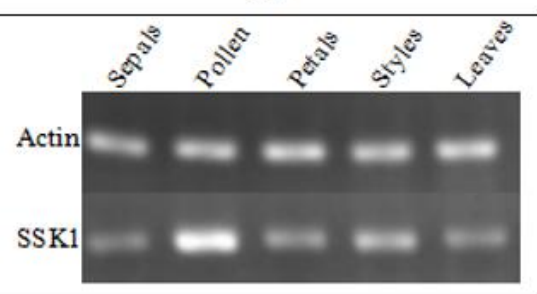

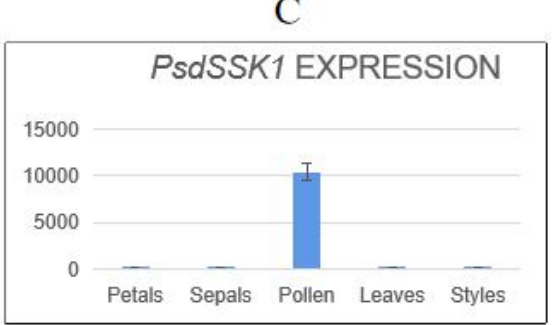

E
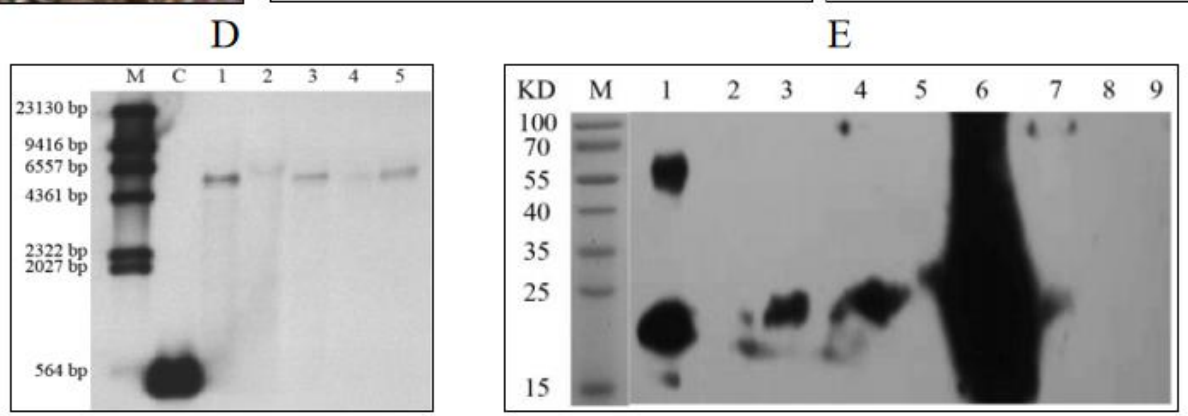

Figure 7 (A) Cultivated almond flowers; (B, C) Differential expression of the PsdSSK1 gene in different tissues of cultivated almond tree; (D) Three hours after exposure to CSPD (Lane M: DNA molecular weight marker; PCR products; Lane 1: Leaves; Lane 2: Petals; Lane 3: Styles; Lane 4: Sepals; Lane 5: Pollen); (E) Western blot detection results (Lane M: Positive control; Lane 2, lane 7, lane 8, lane 9: Leaves; Lane 3: Petals; Lane 4: Styles; Lane 5: Sepals; Lane 6: Pollen)

\subsection{Subcellular localization analysis}

The subcellular location of the PsdSSK1 protein is the cytoplasm. PGC1-PsdSSK1 was transiently transformed into Nicotiana benthamiana epidermal cells. The epidermal cells were removed, and the fluorescence localization was observed under a laser confocal fluorescence microscope; the results are shown in Figure 8. PsdSSK1 was expressed in the cytoplasm, which is consistent with the prediction results of WoLF PSORT online analysis software. 


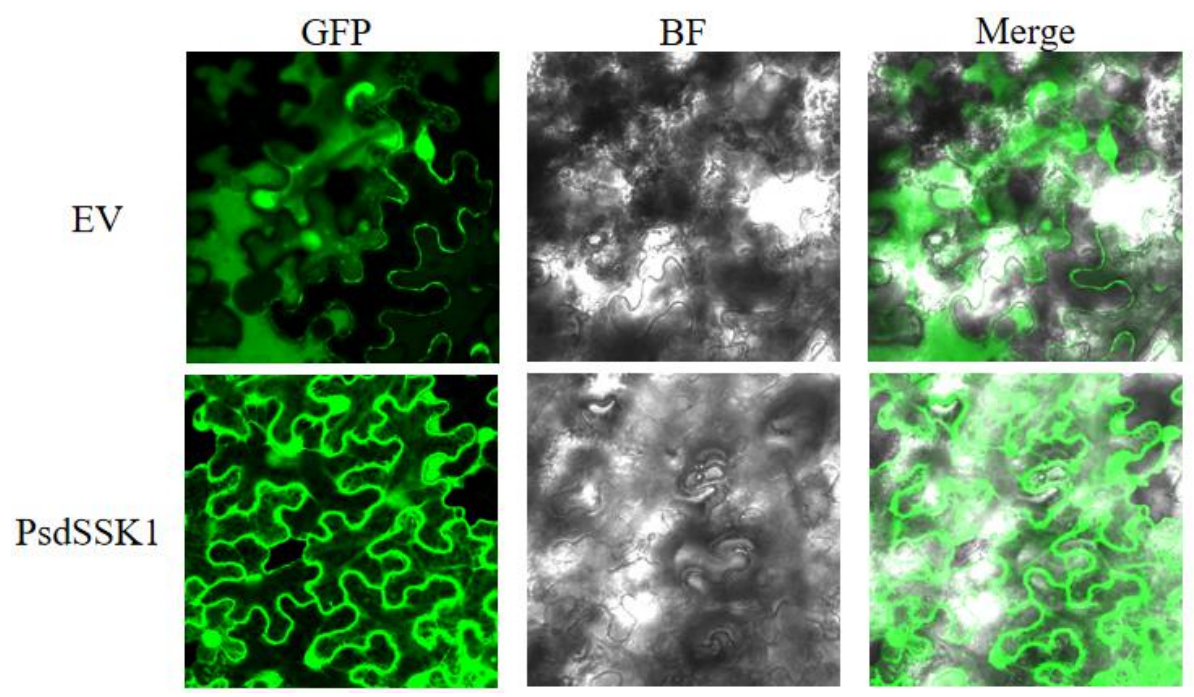

318 Figure 8 Subcellular localization of the PsdSSK1 gene. Green fluorescence (GFP), visible

319 light (BF), merged field (merge), scale bar $=50 \mu \mathrm{m}$.

\section{Discussion}

Skp1 is a component of a SKP1-Cullin1-F-box (SCF) complex that facilitates ubiquitin-mediated protein degradation in eukaryotes. In various plant tissues, the UPP breaks down most intracellular proteins, and the SCF complex is the most common and largest class of E3 family members[32,33]. In this study, we screened 18 PsdSkp1 family members from the almond genome, all of which were acidic and hydrophilic proteins, and their subcellular location was mainly the cytoplasm. The ASK1 and ASK2 proteins in Arabidopsis thaliana exhibit a substantial amount of functional overlap and participate in early growth and the abscisic acid (ABA) signaling pathway[34,35]. Moreover, these proteins have the potential to interact with F-box proteins and participate in SCF complexation[36]. Our phylogenetic tree showed that PsdSkp1-1, PsdSkp1-15, and PsdSkp1-16 strongly clustered together with ASK2. Moreover, the protein interaction results showed that 17 PsdSkp1 members interacted with Cullin and F-box proteins and participated in the formation of the SCF complex (Supplement Figure 1 and Table 2), which suggested that PsdSkpl could regulate the early growth and development of almond and the ABA signaling pathway. GsSKP21 in soybean is highly homologous to ASK20 and $A S K 21$, and its tissue expression characteristics are similar. Overexpression of GsSkp21 in Arabidopsis significantly increased plant tolerance to alkali stress[37]. PsdSkp1-8a, 
PsdSkp1-8b, and PsdSkp1-10 are highly homologous to ASK20 and ASK21, indicating that these three genes may have functions similar to those of GsSKP21, may participate in the ABA signaling pathway and may increase the alkali tolerance of almond. PsdSkp1-12, which may play a role in almond, is highly homologous to SSK1 of 10 species. Subsequent cloning and identification of PsdSSK1 also confirmed this supposition.

The conserved motifs and conserved structures of the proteins revealed that 18 members of PsdSkp1 proteins had Skp1 or Skp1_POZ domains. Studies have shown that all Skpl genes in Arabidopsis and rice originate from the same ancestral gene. Phylogenetic analysis revealed that these genes could be divided into three types: type Ia, which have one intron and two exons; type Ib, which not have introns; and type II, which have many introns[38]. The lack of introns in the Skpl gene may be caused by reverse transcriptase-mediated rearrangement[38]. Repeated signals and poly(A) extensions of target sites were found in the rice OSK3 sequence, which may be caused by the rapid evolution of these genes, as shown in both rice and Arabidopsis[39]. According to the phylogenetic tree clustering, the exon-intron data showed that the PsdSkpl family members were highly conserved, and the exon-intron differences among different groups were substantial. Eight PsdSkpl family members did not contain introns, which corresponded to the results obtained in Arabidopsis and rice. The SKP1 gene in dicotyledonous plants and monocotyledonous plants evolved from a single ancestral gene, followed by multiple duplication events[40]. These gene duplication events are essential for the evolution of gene families and provides a good basis for the evolution of new genes as well as for new physiological and morphological changes[41]. In addition, the PsdSSK1 family members resulting from fragment duplication and tandem repeats may provide new functions for almond Skp1 members and promote the growth and development of almond. The collinear gene pairs of almond, Arabidopsis thaliana, Malus domestica, Prunus persica and Prunus mume were mainly purified, and the gene functions were highly evolutionarily conserved. The promoter region annotations revealed that PsdSkpl contains many kinds of elements, such as those that respond to hormones, stress, growth and development, that play an important role in overall plant growth and development. 
In Arabidopsis, the $\beta$-glucuronidase (GUS) activity of $A S K s$ occurs mainly in anthers and stigmas[42], and $A S K s$ have been shown to play an important role in flower development and male gametophyte sterility[11]. Most PsdSkpl proteins were expressed in flower buds and pistils. Interestingly, the genes expressed in the flower buds have two or more exons and one or more introns; in the pistils, PsdSkp1-7, PsdSkp1-11, PsdSkp1-12 and PsdSkp1-16 contain only one exon and 0 exons. These results indicate that the number of introns and exons is closely related to the expression activity of PsdSkpl in different parts of floral organs. $P s d S k p 1-7$ and $P s d S k p 1-12$ were significantly highly expressed in the control anthers, and the expression of PsdSkp1-6 was significant in anthers under freezing stress. Compared with PsdSkp1-7 and PsdSkp1-12, PsdSkp1-6 has one more MYB transcription factor-binding site (MBSI), which may regulate the production of flavonoids to make anthers resist freezing stress[43]. In the control ovaries, the expression of PsdSkp1-1, PsdSkp1-3, PsdSkp1-4, PsdSkp1-8a, PsdSkp1-8b and PsdSkp1-10 genes was significant, which was inhibited after freezing stress, while the expression of PsdSkp1-13, PsdSkp1-15, PsdSkp1-16 and PsdSkp1-17 genes was significant. In AXA self-pollination, PsdSkp1-1, PsdSkp1-3, PsdSkp1-10 and PsdSkp1-16 were highly expressed. Notably, the expression of these four genes in AXA changed little, which may be due to the expression in the pistil itself. It is suggested these genes may not be expressed or that their products may not have any activity in pollen. After IXA cross-pollination, the expression of PsdSkp1-8a, PsdSkp1-12, PsdSkp1-13 and PsdSkp1-15 decreased significantly (or not) in AXA. Almond is a typical self-incompatible plant species, with abnormal self-pollination that leads to loss of pollen activity; however, cross-pollination is normal, and pollen remains active. These results indicate that these four genes regulate pollen expression and promote cross-pollination. In summary, PsdSkpls and ASKs have similar functions and play an important role in the growth and development of flower organs and male gametophyte sterility. In addition, the regulation of pollen expression by $P S d S k p 1$ proteins is severely affected by freezing stress, and cross-pollination may not be normal under freezing conditions. 
SSK1 can be used as a component to form a functional SCF complex together with Cullin1, SLF and RBX1. At present, SSK1 genes have been identified in apple, petunia and other species[44,45]. SSK1s constitute a new class of Skp1-like proteins. The amino acids of AhSSK1 and PhSSK1 are 40\% homologous with those of other Skp1 proteins, and both have a unique C-terminus comprising 7-9 amino acid residues, which is similar to the conventional WAFE terminal residues found in most plant Skp1 homologs[44]. ORF analysis of the cloned PsdSSK1 gene revealed that the gene is 534 bp in length, encoding 178 amino acids. Multiple alignment of the sequence of the SSK1 protein with sequences from other species revealed two distinct domains: Skp1_POZ and Skp1. The quantitative fluorescence expression results showed that PsdSSK1 was highly expressed in almond pollen, which was consistent with the specific expression of the SSK1 gene in pollen. Southern blot analysis of the PsdSSK1 gene revealed present as single copy in the leaves, petals, style, sepals and pollen. The Western blot results showed that the PsdSSK1 protein was expressed in the petals, styles, and pollen tissues of almond, and the expression of PsdSSK1 in the pollen was significantly higher than that in the other tissues. The subcellular location of the PsdSSK1 protein was consistent with the results predicted by WoLF PSORT online analysis software, which was predicted to localize to the cytoplasm. Interestingly, PsdSSK1 and PsdSkp1-12 were identical. Combining the phylogenetic tree results, expression patterns and experimental results, we found that $P s d S k p 1-12$ was an ssk1 gene in almond that could replace the Skpl gene and bind to SLF and cullin1. PsdSkp1-12 may form a functional SCF complex together with cullin1, SLF and Rbx1 as components to regulate almond pollen activity, SI, and cross-compatibility.

\section{Materials and Methods}

4.1 Screening of the $S k p 1$ gene family members in almond and analysis of the physicochemical properties of their proteins

The coding DNA sequence (CDS) of D. almondi was downloaded from the Genome Database for Rosaceae (https://www.rosaceae.org/), and the hidden Markov model (HMM) profiles of Skp1 and Skp1_POZ (accession numbers PF01466 and PF03931) were downloaded from the Pfam database (http://pfam.xfam.org/). The HMMsearch tool was used to search and compare the whole-genome protein data of almond with data of the Skp1 
and Skp1_POZ hidden Markov models[46,47]. Domain verification was performed through the Pfam database, and Skp1 protein motifs and redundant genes were discarded. Finally, 18 Skpl genes were obtained.

\subsection{Sequence alignment, phylogenetic analysis, chromosomal location analysis, gene structure} construction, collinearity analysis, and calculation of $\mathrm{Ka}$ and $\mathrm{Ks}$ values

The protein sequences of the $18 S k p l$ genes from almond were analyzed to determine the number of amino acids, protein molecular weight, isoelectric point and hydrophobicity of the protein, and ExPASy (http://au.expasy.org/tools) and WoLF PSORT II (https://www.genscript.com/wolf-psort.html?src=leftbar) were used for protein subcellular localization analysis[48,49]. Multiple alignment of the nucleotide and amino acid sequences were performed using ClustalW[50]. Phylogenetic analysis was performed based on the sequences of SSK1 proteins from Antirrhinum hispanicum, Petunia hybrida, Prunus persica, Prunus tenella, Prunus avium, Malus domestica, Pyrus ussuriensis x Pyrus communis, Fragaria ananassa, Populus alba and Jatropha curcas via the maximum likelihood method with 1000 bootstrap replicates and visualized with MEGA X software[51]. The chromosomal distribution of all PsdSkpl genes was determined based on the identification results, and images of the locations of PsdSkpl genes were subsequently drawn with TBtools software. Exon-intron structure analysis of the PsdSkpl genes was conducted and the results displayed by comparing CDSs and their corresponding gene sequences from the genome using TBtools[52]. The MEME program was used to statistically identify conserved motifs within the complete amino acid sequences of the PsdSkp1 proteins[53]. Gene duplication and collinearity analyses were performed using MCScanX via GFF3 files retrieved from the Phytozome database[54]. Genes that were not present on major chromosomes were excluded from the evaluation. Collinearity circle plots were generated using Circos v0.69[55], and the KaKs_Calculator tool was used to calculate the $\mathrm{Ka} / \mathrm{Ks}$ values of the collinear genes[56].

\subsection{Analysis of cis-elements within $S k p 1$ gene members}

The 2000 bp upstream sequences of the identified $S k p 1$ genes were extracted by TBtools[57]. The extracted sequences were then submitted to the PlantCARE website (http://bioinformatics.psb.ugent.be/webtools/plantcare/html/) to predict the cis-elements in 
the promoter regions. A diagram of the cis-elements within the $S k p 1$ genes was constructed by TBtools and modified via Adobe Illustrator CC 2019.

\subsection{Analysis of the expression patterns of members of the $S k p 1$ family in almond}

Sequences from following almond tissues were downloaded from the NCBI Sequence Read Archive (SRA) database: leaves (SRR12806975); flower buds (SRR11251344); pistils (SRR5830017); fruitlets (SRR10189226); anthers under normal and freezing-stress conditions (control: SRR2976058; freezing stress: SRR2976061); ovaries under normal and freezing-stress conditions (control: SRR2976060; freezing stress: SRR2976062); and pollen from selfing and outcrossed unpollinated A2-198 pistils (A) (ERR2182599), unpollinated ITAP-1 pistils (I) (ERR2182601), self-pollinated A2-198 pistils (AxA) (ERR2182600), and ITAP-1 pistils pollinated with A2-198 (IxA) (ERR2182602). The transcriptome data were obtained, and FPKM values were subsequently calculated. Afterward, a heat map of the expression patterns of the $18 \mathrm{Skpl}$ family members was constructed.

\subsection{Cloning of the PsdSSK1 gene}

Tissue samples of leaves, petals, sepals, styles, and pollen of cultivated almond trees were collected and then quickly and completed ground into a powder in a sterile mortar filled with ERR 2182602 liquid nitrogen. Total RNA was extracted using a polysaccharide polyphenol kit from Tiangen, and the integrity of the total RNA was verified via 1\% agarose gel electrophoresis[58]. First-strand cDNA was subsequently synthesized using a Fermentas RevertAid First Strand cDNA Synthesis Kit. According to the sequences of the conserved regions of the homologous genes of the target gene available in public databases, a pair of degenerate primers was designed using Primer Premier 5.0 software. PCR amplification of the PsdSSK1 gene was then performed in which cDNA was as a template. The PCR conditions were as follows: $94{ }^{\circ} \mathrm{C}$ predenaturation for $2 \mathrm{~min}$; 35 cycles of $94{ }^{\circ} \mathrm{C}$ for $30 \mathrm{~s}, 55{ }^{\circ} \mathrm{C}$ for $30 \mathrm{~s}$, and $72{ }^{\circ} \mathrm{C}$ for $30 \mathrm{~s}$; a $72{ }^{\circ} \mathrm{C}$ extension for $10 \mathrm{~min}$; and storage at $4{ }^{\circ} \mathrm{C}$. The PCR products were analyzed via agarose gel electrophoresis and recovered with a spin column-type agarose gel DNA recovery kit. The PCR product was ligated to a pMD-18 vector cloning vector using a T4 ligase kit from Takara. The ligation product was transformed into Escherichia coli $\mathrm{DH} 5 \alpha$ competent cells via the heat-shock method $\left(42^{\circ} \mathrm{C}\right.$ for $45 \mathrm{~s}$ ). After overnight culture at $37^{\circ} \mathrm{C}$, positive monoclonals were selected and sent to the company for sequencing. 
Five tissue samples of cultivated almond were ground in a mortar with TRIzol reagent and incubated at room temperature for $5 \mathrm{~min}$. Afterward, chloroform was added for extraction for $3 \mathrm{~min}$, after which the mixture was centrifuged at $12000 \times \mathrm{g}$ at $4{ }^{\circ} \mathrm{C}$ for 15 min. The top colorless liquid was removed and isopropyl alcohol was added to precipitate the RNA. Afterward, the mixture was incubated at room temperature for $10 \mathrm{~min}$. It was then centrifuged at $12000 \times \mathrm{g}$ for $10 \mathrm{~min}$ at $4{ }^{\circ} \mathrm{C}$, after which $75 \%$ ethanol in diethyl phosphorocyanidate (DEPC) water was used to clean the RNA. The ethanol was removed by centrifugation, after which the RNA pellet was dried and dissolved in DEPC water. The integrity of the RNA was detected by $1.2 \%$ agarose gel electrophoresis[59,60]. A RevertAid First Strand cDNA Synthesis Kit from Thermo Scientific was used to synthesize first-strand cDNA, which was stored at $-20^{\circ} \mathrm{C}$ until use. Using the diluted cDNA as a template and PsdSSK1-F and PsdSSK1-R as upstream and downstream primers, respectively, almond $\beta$-actin was selected as an internal reference gene, and actin-F and actin-R were selected as internal reference gene primers. Real-time PCR was subsequently performed. The reaction system $(25 \mu \mathrm{L})$ consisted of the following: $10 \mu \mathrm{L}$ of SYBR ${ }^{\circledR}$ Green PCR Master Mix, $0.4 \mu \mathrm{L}$ of forward primer $(10 \mu \mathrm{mol} / \mathrm{L}), 0.4 \mu \mathrm{L}$ of reverse primer $(10$ $\mu \mathrm{mol} / \mathrm{L}), 5 \mu \mathrm{L}$ of diluted cDNA $(50 \mathrm{ng} / \mu \mathrm{L})$, and $25 \mu \mathrm{L}$ of RNase-free water[61]. The reaction conditions were as follows: predenaturation at $95^{\circ} \mathrm{C}$ for $3 \mathrm{~min} ; 40$ cycles of $95^{\circ} \mathrm{C}$ for $10 \mathrm{~s}, 55^{\circ} \mathrm{C}$ for $20 \mathrm{~s}$, and $72^{\circ} \mathrm{C}$ for $20 \mathrm{~s}$; and then $75^{\circ} \mathrm{C}$ for $5 \mathrm{~s}$. Plate reads were added to collect fluorescence signals (40 cycles). The melting curve analysis temperature range was $65 \sim 95{ }^{\circ} \mathrm{C}$, and the temperature was increased by $0.5^{\circ} \mathrm{C} / 5 \mathrm{~s}$. Three biological replicates were included per sample, three technical replicates were included for each biological replicate, and the program was run after all settings were saved. The obtained cycle threshold (CT) value was quantitatively analyzed via the $2_{-}^{\triangle}{ }^{\triangle} \mathrm{CT}$ method[62].

\subsection{Southern blot hybridization}

By using a cetyl-trimethylammonium bromide (CTAB) plant genomic DNA rapi d extraction kit from Zhongding Company to extract genomic DNA from five tissue $\mathrm{s}$ of cultivated almond trees, we performed a single digestion of the genome by the use of the restriction enzyme EcoRI. The digested DNA was separated via $0.7 \%$ a garose gel electrophoresis at $25 \mathrm{~V}$ (constant) and $4^{\circ} \mathrm{C}$ overnight. The DNA was the $\mathrm{n}$ transferred to a positively charged HyBond $\mathrm{N}+$ nylon membrane via an upward $\mathrm{c}$ apillary method, after which the membrane was transferred for approximately $20 \mathrm{~h}$, heat, and fixed at $80^{\circ} \mathrm{C}$ for $2 \mathrm{~h}[63]$. Roche's Digoxin Hybridization Detection Kit II 
(chemiluminescence method) was used to synthesize probes and design primers bas ed on the sequence of the PsdSSK1 gene. PsdSSK1-F (5'-AGGAGGAGAAGAACGA CG-3') and PsdSSK1-R (5'-TTCAAAAGCCCAAGCATA-3') were used as the forwar $\mathrm{d}$ and reverse primers, respectively, and the hybridization probe was amplified and $\mathrm{s}$ ynthesized via PCR . The PCR conditions were as follows: $94^{\circ} \mathrm{C}$ predenaturation for $5 \mathrm{~min} ; 35$ cycles of $94^{\circ} \mathrm{C}$ denaturation for $30 \mathrm{~s}, 55^{\circ} \mathrm{C}$ annealing for $30 \mathrm{~s}$, and $72^{\circ}$ $\mathrm{C}$ extension for $40 \mathrm{~s}$; and $72^{\circ} \mathrm{C}$ extension for $7 \mathrm{~min}$. The PCR product obtained aft er labeling with digoxin was analyzed via 1\% agarose gel electrophoresis and recov ered by an agarose gel purification recovery kit[64]. The cells were subsequently pr ehybridized at $37^{\circ} \mathrm{C}$ for $2 \mathrm{~h}$, depleted of the prehybridization solution, added to the hybridization solution containing a digoxin-labeled probe, and hybridized overnight at $37^{\circ} \mathrm{C}[65]$. After hybridization, the membrane was washed twice with $20 \mathrm{~mL}$ of $2 \times$ SSC and $0.1 \%$ SDS solution for $5 \mathrm{~min}$ each at room temperature. The membrane was then washed twice with $20 \mathrm{~mL}$ of $1 \times \mathrm{SSC}$ and $0.1 \%$ SDS solution at $65^{\circ} \mathrm{C}$ for 15 min each time. The membrane was then equilibrated in $20 \mathrm{~mL}$ of washing buff er for 2-5 min, after which it was placed in $10 \mathrm{~mL}$ of blocking solution for $30 \mathrm{mi}$ n. Afterward, $1 \mathrm{~mL}$ of CSPD was added to the front side (nucleic acid side) of the membrane, which was then air dried at $15-25^{\circ} \mathrm{C}$ for $5 \mathrm{~min}$. The excess liquid was removed, after which the membrane was incubated at $37^{\circ} \mathrm{C}$ for $10 \mathrm{~min}$ before mac hine exposure; the experimental results were recorded[66].

\subsection{Western blot hybridization}

Samples of leaves, petals, styles, sepals, and pollen tissues of almond trees cultivated in Xinjiang were sampled by the bicinchoninic acid assay (BCA) method. After loading, the polyacrylamide gel was run at $100 \mathrm{~V}$, and the voltage was increased to $160 \mathrm{~V}$ until electrophoresis was completed[67]. The gel was removed and transferred to a membrane with a constant current of $280 \mathrm{~mA}$ for approximately $1 \mathrm{~h}$. After electroporation was completed, the membrane was removed and washed 4 times with poly(butylene succinate-co-terephthalate) (PBST) for 5 min each. The membrane was blocked in a 5\% skim milk powder blocking solution at $37^{\circ} \mathrm{C}$ for $1 \mathrm{~h}$. A primary antibody was then diluted 1:500 with PBST, and the membrane was incubated at $4^{\circ} \mathrm{C}$ overnight in the primary antibody dilution[68]. After removing the membrane the next day, the membrane was washed 4 times with PBST for 5 min each time. A secondary antibody was diluted with a blocking solution containing 5\% skim milk powder at a ratio of 1:5000. The membrane was allowed to react at $37^{\circ} \mathrm{C}$ for $1 \mathrm{~h}$ with the secondary antibody. After the reaction was 
completed, the membrane was removed, and it was washed 4 times in a clean box for 5 min each. Enhanced chemiluminescence (ECL), development, and exposure were then performed[69].

\subsection{Construction and subcellular location of the PsdSSK1 fusion expression vector}

The subcellular location of the PsdSSK1 protein was predicted using the WoLF PSORT II tool. According to the sequencing results and primer design principles of in-fusion construction technology, specific primers (PsdSSK1-GFP-F:GGAATTCATGTCGGCCGAGGAGGAGA and PsdSSK1-GFP-R:CCCAAGCTTGTCCTCATCAACTCCTTCAA) with 17 bp vector sequences were designed. The bacterial fluid plasmid containing the correct sequencing results of a pTOPO vector containing the target gene was used as a template, and the reaction system was the same as that used in Section 3.2. Gene sequences containing enzyme restriction sites were amplified via PCR. A PGC-1 vector was digested with the restriction enzymes EcoRI and XHOI to obtain a linearized PGC-1 vector. With in-fusion HD Enzyme Premix, the target gene fragment was inserted into the linearized PGC-1 vector to obtain a PGC1-PsdSSK1 expression vector. The specific operation process was performed according to the instructions of the Novozyme In-Fusion reagent. The plasmid construct was verified via bacterial liquid PCR and sequencing, and the expected positive clones were obtained. The pGC1-PsdSSK1 fusion expression vector construct was transferred into competent Agrobacterium tumefaciens GV3101 cells by the freeze-thaw method and then coated in YEB medium that included kanamycin and rifampicin. Monoclones were selected and verified via bacterial liquid PCR, and bacterial liquid containing the correct bands was selected for propagation to an OD600 of 1.0. The samples were subsequently centrifuged at $4000 \times \mathrm{g}$ for $10 \mathrm{~min}$ to collect the thalli. The supernatant was then discarded with resuspended buffer solution $(10 \mathrm{mmol} / \mathrm{L}$ 2-(N-morpholino)ethanesulfonic acid (MES), $10 \mathrm{mmol} / \mathrm{L} \quad \mathrm{MgCl}_{2}, 150 \mu \mathrm{mol} / \mathrm{L} \quad \mathrm{A}$ ), resuspended to an OD600 of 1.0, and incubated at room temperature for 3-4 h. Young and tender tobacco leaves in a good growth state were selected, and the resuspension was carefully injected with a sterile injector into the abaxial side of the leaves for infection. After 2 days of culture, the results were observed by laser confocal microscopy. 
performed the analysis. Jiahui Yang, Wenwen Gao, Yilian Tang and Xintong Ma participated in sample collection and conducted experiments. All authors read and approved the final manuscript.

Funding: This work was supported by "Xinjiang Uygur Autonomous Region Natural Science Foundation" (Grant Number 2019D01A56), “The Key Disciplines Project of Horticulture of Xinjiang Uygur Autonomous Region” (Grant Number 2016-10758-3).

\section{Abbreviations}

Psd Prunus dulcis

GRAVY Grand average of hydropathicity

SI Self-incompatibility

Skp1_POZ S-phase kinase-associated protein 1, tetramerisation domain

UTR Untranslated region

aa Amino acid

bp Base pair

Ka Non-synonymous substitution

Ks Synonymous substitution

\section{ABA Abscisic acid}

\section{References}

1. Parry,G.; Estelle,M.;Auxin receptors.A new role for F-box proteins.Curr. Opin.Cell Biol.2006, 18,152-156.

2. Ni,W.; Xie,D.;Hobbie,L.;Feng,B.;Zhao,D.;Akkara,J.;Ma,H.Regulation of Flower Development by SCF Complexes1 in Arabidopsis. Plant Physiol. 2004,134,1574-1585.

3. Liu,F.; Ni,W.;Griffith,M.E.; Huang,Z.; Chang,C.; Peng,W.; Ma,H.; Xie,D.The ASK1 and ASK2 Genes Are Essential for Arabidopsis Early Development. Plant Cell 2004, 16, 5-20.

4. Kong,H.; Leebens-Mack,J.; Ni,W.; DePamphilis,C.W.; Ma,H.Highly Heterogeneous Rates of Evolution in the SKP1 Gene Family in Plants and Animals: Functional and Evolutionary Implications. Mol. Biol. Evol. 2004, 21, $117-128$.

5. Elzanati,O.;Roche,J.;Boulaflous-Stevens,A.;Mouzeyar,S.;Bouzidi,M.F.Genome-wide.analysis,classification, expression and interaction of Physcomitrella patens SKP1-like (PpSKP) and F-box (FBX) genes. Plant Gene 2017,12,13-22.

6. Kahloul,S.; Hajsalah El Beji, I.; Boulaflous, A.; Ferchichi, A.; Kong, H.; Mouzeyar, S.; Bouzidi, M.F. Structural,expression and interaction analysis of rice SKP1-like genes. DNA Res. 2013,20, 67-78.

7. Mason Bethany,Laman Heike. The FBXL family of F-box proteins: variations on a theme.Open biology,2020,10(11),200319.

8. Xu,L.; Liu, F.; Lechner, E.; Genschik, P.; Crosby, W.L.; Ma, H.;Peng,W.; Huang, D.; Xie, D. The SCF ${ }^{\text {CoI1 }}$ ubiquitin-ligase complexes are required for jasmonate response in Arabidopsis.Plant Cell.2002,14,1919-1935.

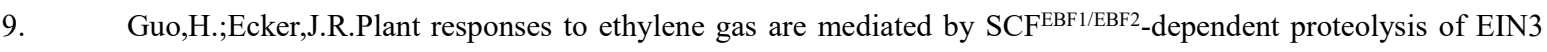


transcription factor. Cell, 2003,115, 667-677.

10. Ni,W.;Xie,D.;Hobbie,L.;Feng,B.;Zhao,D.;Akkara,J.;Ma,H.Regulation of flower development in Arabidopsis by SCF complexes. Plant Physiol. 2004,134, 1574-1585.

11. Wang,Y.; Yang,M.The ARABIDOPSIS SKP1-LIKE1 (ASK1) protein acts predominately from leptotene to pachytene and represses homologous recombination in male meiosis.Planta, 2005,223, 613-617.

12. Liu,F.;Ni,W.;Griffith, ME.; Huang, Z.;Chang, C.;Peng, W.;Ma, H.; Xie, D.; The ASK1 and ASK2 genes are essential for Arabidopsis early development. Plant Cell.2004,16:5-20.

13. Zhao,L.;Huang,J.;Zhao,ZH.;Li,Q.;Sims,TL.;Xue,YB.The SKP1-like protein SSK1 is required for cross-pollen compatibility in S-RNase-based self-incompatibility. Plant J 2010,62:52-63.

14. Marrocco,K.;Lecureuil ,A.;Nicolas,P.; Guerche, P, The Arabidopsis SKP1-like genes present a spectrum of expression profiles. Plant Mol Biol .2003,527:15-727.

15. Zhang,Y.Q.;Wang,C.P.;,Lin,Q.F.;Gao,F.H.;Ma,Y.;Zhang,M.;Lin,Y.H.;Ma,Q.H.;Hua,X.J,Genome-wide analysis of phylogeny, expression profile and sub-cellular localization of SKP1-Like genes in wild tomato. Plant Science,2015,238.105-114.

16. Min,J.H.;Dae,Y.K.;Seo,Y.W, SKP1-like-related genes interact with various F-box proteins and may form SCF complexes with Cullin-F-box proteins in wheat.Molecular Biology Reports,2013,40(2).969-981.

17. Hao,Q.;Ren,H.X.;Zhu,J.;Wang,S.C.;Liu,H.Z.;Gao,Z.;Shu,Q.Y, Overexpression of PSK1,a SKP1-like gene homologue, from Paeonia suffruticosa ,confers salinity tolerance in Arabidopsis. Plant Cell Reports,2017,36(1).151-162.

18. Wang,X.;Ni,W.;Ge,X.;Zhang,J.;Ma,H.;Cao,KM,Proteomic identification of potential target proteins regulated by an ASK1-mediated proteolysis pathway. Cell Res,2006,16,489-498.

19. Hu,D,L.;Chen,Q.Z.;Zhang,C.J.;Wang,Y.;Zhang,B.J.;Tang,C.M.;Identification of cotton SKP 1-like gene GhSKP1 and its function in seed germination and taproot growth in tobacco. Canadian Journal of Plant Science,2013,93(5),817-825.

20. Chen,Y.;Xu,Y.;Luo,W.;Li,W.;Chen,W.;Zhang,D.;Chong,K.;The F-box protein OsFBK12 targets OsSAMS1 for degradation and affects pleiotropic phenotypes,including leaf senescence, in rice, Plant Physiol. 2013,163,1673-1685.

21. Huang,J.;Zhao,L.AhSSK1, a novel SKP1-like protein that interacts with the S-locus F-box protein SLF.The Plant Journal.2006.46(5):780-793.

Zhao,L.;Huang,J.;Zhao,Z,The Skp1-like protein SSK1 is required for cross-pollen compatibility in S-RNase-based self-incompatibility. The Plant Journal. 2010,62(1),52-63.

23. Xu C.;Li M.;Wu J,Identification of a canonical SCF(SLF)complex involved in S-RNase-based self-incompatibility of Pyrus(Rosaceae). Plant Molecular Biology. 2012,81(3), 245-257.

24. Minamikawa,MF.;Koyano R.;Kikuchi S,Identification of SFBB-Containing Canonical and Noncanonical SCF Complexes in Pollen of Apple (Malus $\times$ domestica). PLoS ONE. 2014, 9(5),e97642.

25. Claessen,H.;Keulemans,W.;Poel,BVD,Finding a Compatible Partner: Self- Incompatibility in European Pear (Pyrus communis); Molecular Control, Genetic Deter-mination, and Impact on Fertilization and Fruit Set. Frontiers in plant science. 2019,10,407.

Zeng,B.;Wang,J.Y.;Hao,Q.;Identification of a Novel SBP1-Containing SCF ${ }^{\mathrm{SFB}}$ Complex in Wild Dwarf Almond (Prunus tenella). Front.Genet. 2019,10,1019.

27. Fujii,S.;Shimosato-Asano,H.;Kakita,M.Parallel evolution of dominant pistil-side self-incompatibility suppressors in Arabidopsis. Nature Communications. 2020,11(1). 
29. Mathieu,G.;Vincent,C.;Xavier,V.Genotyping and De Novo Discovery of Allelic Variants at the Brassicaceae Self-Incompatibility Locus from Short-Read Sequencing Data.Molecular Biology and Evolution, 2019(4).4.

30. McClure,B.;Cruz-Garcia,F.;Romero,C.Compatibility and incompatibility in S-RNase-based systems. Ann Bot. 2011,108,647-658.

31. Schulman,B.A.; Carrano,A.C.;Jeffrey,P.D. Insights into scf ubiquitin ligases from the structure of the skp1-skp2 complex. Nature. 2000,408(6810),381-386.

32. Zheng,N.;Schulman,B.A.;Song,L. Structure of the Cul1-Rbx1-Skp1-F boxSkp2 SCF ubiquitin ligase complex. Nature. 2002,416(6882),703-709.

33. Fernándezi,Martí,A.;Gradziel,T.M.;Sociasi Company R.Methylation of the S F-locus in almond is associated with S-RNase loss of function. Plant Molecular Biology.2014,86(6),681-689.

34. Risseeuw,E.P.;Daskalchuk,T.E,Banks,T.W.;Liu,E.;Cotelesage,J.Protein interaction analysis of SCF ubiquitin E3 ligase subunits from Arabidopsis. Plant J 2003,34: 753-767.

35. Liu,F.;Ni,W.;Griffith,M.E.;Huang,Z.;Chang,C.The ASK1 and ASK2 genes are essential for Arabidopsis early development. Plant Cell.2004,16: 5-20.

36. Wei Jianxin,Dong Su,Yao Kangning,Martinez Maria Francesca Ysabelle M,Fleisher Paine R,Zhao Yutong,Ma Haichun,Zhao Jing. Histone acetyltransferase CBP promotes function of SCF FBXL19 ubiquitin E3 ligase by acetylation and stabilization of its F-box protein subunit.. FASEB journal : official publication of the Federation of American Societies for Experimental Biology,2018,32(8):fj.201701069R.

37. Liu,A.;Yu,Y.; Duan,X. GsSKP21, a Glycine soja S-phase kinase-associated protein, mediates the regulation of plant alkaline tolerance and ABA sensitivity.Plant Molecular Biology,2015, 87(1-2),111-124.

38. Kong,H.;Landherr,L.L.;Frohlich,M.W.Patterns of gene duplication in the plant SKP1 gene family in angiosperms: evidence for multiple mechanisms of rapid gene birth.Plant Journal, 2010, 50(5),873-885.

39. Kong,H.;Jim L,M.;Ni,W. Highly Heterogeneous Rates of Evolution in the SKP1 Gene Family in Plants and Animals:Functional and Evolutionary Implications.Molecular Biology \& Evolution, 2004(1),117-128.

40. Horan,K.;Lauricha,J.;Bailey-Serres,J.;Raihhel,N.;Girke,T.Genome cluster database: a sequence family analysis platform for Arabidopsis and rice. Plant Physiol.2005,138,47-54.

41. Maere,S.;DeBodt,S.;Raes,J.;Casneuf,T.;Van,Montagu,M.;Kuiper,M.;Van,de.;Peer,Y. Modeling gene and genome duplications in eukaryotes.Proc.Natl Acad.Sci.USA,2005,102, 5454-5459.

42. Takahashi,N.;Kuroda,H.;Kuromori,T.;Hirayama,T.;Seki,M.;Shinozaki,K.;Shimada,H.;Matsui,M,Expression and interaction analysis of Arabidopsis Skp1-related genes.Plant Cell Physiol,2004,45,83-91.

43. Schulz,E.;Tohge,T.;Zuther,E,Flavonoids are determinants of freezing tolerance and cold acclimation in Arabidopsis thaliana. Scientific Reports, 2016, 6,34027.

44. Hidenori,S,Molecular mechanism of the S-RNase-based gametophytic self-incompatibility in fruit trees of Rosaceae. Breeding Science, 2016, 66(1),116-121.

45. Yuan,H.;Meng,D.;Gu,Z.A novel gene, MdSSK1, as a component of the SCF complex rather than MdSBP1 can mediate the ubiquitination of S-RNase in apple. Journal of Experimental Botany. 2014, 65(12),3121-3131.

46. Finn,R.D.;Coggill,P.;Eberhardt,R.Y.; Eddy, S.R.; Mistry, J.; Mitchell, A.L.; Potter, S.C.; Punta, M.;Qureshi,M.;Sangrador-Vegas,A.The Pfam protein families database: Towards a more sustainable future. Nucleic Acids Res. 2016, 44, D279-D285.

47. Mistry,J.;Finn,R.D.;Eddy,S.R.;Bateman,A.;Punta,M.Challenges in homology search: HMMER3 and convergent evolution of coiled-coil regions. Nucleic Acids Res. 2013, 41, e121.

48. Gasteiger,E.;Gattiker,A.;Hoogland,C.;Ivanyi,I.;Appel,R.D.;Bairoch,A,ExPASy:the proteomics server for in-depth protein knowledge and analysis. Nucleic Acids Res ,2003,31,3784-3788.

49. Paul,H.;Keun-Joon,P.;Takeshi,O.WoLF PSORT: protein localization predictor. Nucleic Acids Research, 2007, 
35(Web Server issue):585-587.

50. Hung,J.H.;Weng,Z.P.Sequence Alignment and Homology Search with BLAST and ClustalW. Cold Spring Harbor protocols,2016(11).

51. Kumar,S.;Stecher,G.;Tamura,K.;MEGA7:molecular evolutionary genetics analysis version 7.0 for bigger datasets. Mol Biol Evol. 2016,33(7),1870-4.

52. Chen,C.;Chen,H.;Y Zhang,TBtools:An Integrative Toolkit Developed for Interactive Analyses of Big Biological Data. Molecular Plant, 2020, 13(8).

53. Bailey,T.L.;Mikael,B.;Buske,F.A,MEME Suite: tools for motif discovery and searching.Nucleic Acids Research, 2009, 37(Web Server issue),W202-W208.

54. Wang,Y.;Tang,H.;Debarry,J.D,MCScanX: a toolkit for detection and evolutionary analysis of gene synteny and collinearity. Nucleic Acids Research, 2012, 40(7),e49-e49.

55. Krzywinski,M.;Schein,J.;Birol,I.Circos: An information aesthetic for comparative genomics. Genome Research, 2009, 19:1639-1645.

56. D,Wang.;Zhang,Y.;Zhang,Z.;KaKs_Calculator 2.0:A Toolkit Incorporating Gamma-Series Methods and Sliding Window Strategies.Genomics,Proteomics\& Bioinformatics,2010, 8(1),77-80.

57. Lescot, M,PlantCARE, a database of plant cis-acting regulatory elements and a portal to tools for in silico analysis of promoter sequences. Nucleic Acids Research, 2002, 30(1):325-327.

58. Willison,L.N.;Tripathi,P.;Sharma,G,Cloning,Expression and Patient IgE Reactivity of Recombinant Pru du 6, an 11S Globulin from Almond.International Archives of Allergy and Immunology. 2011,156,3,267-281.

59. Lee,C.;Kim,J.;Shin,S.G.Absolute and relative QPCR quantification of plasmid copy number in Escherichia coli. Journal of Biotechnology. 2006,123(3), 273-280.

60. Stålberg,A.;Kubista,M.The workflow of single-cell expression profiling using quantitative real-time PCR. Expert Rev. Mol. Diagn. 2014,14(3),323-331.

61. Mestdagh,P,Van Vlierberghe P,De Weer,A.A novel and universal method for microRNA RT-qPCR data normalization Genome Biology. 2009; 10(6): R64.

62. Moraes,G.P.;Benitez,L.C.;Amaral,M,Evaluation of reference genes for RT-qPCR studies in the leaves of rice seedlings under salt stress.Genetics \& Molecular Research Gmr,2015, 14(1):2384-98.

63. Cai,D.;Nie,H.;Yan,R.A Southern Blot Assay for Detection of Hepatitis B Virus Covalently Closed Circular DNA from Cell Cultures. Methods in Molecular Biology. 2013,1030,151-161.

64. Aravalli,R.N.;Park,C.W.;Steer,C.J.;Detection of Sleeping Beauty transposition in the genome of host cells by non-radioactive Southern blot analysis. Biochemical and Biophysical Research Communications. 2016,477(3),317-321.

65. Bortoloto,T.M.;Fuchs-Ferraz,MCP.;Kettener,K,Identification of a molecular marker associated with lignotuber in Eucalyptus ssp. Scientific Reports. 2020,10(1).

66. Zhang,B.;Liu,Z.;Ma,J.;Alternative splicing of the AGAMOUS orthologous gene in double flower of Magnolia stellata (Magnoliaceae). Plant Science. 2015,241, 277-285.

67. Liu,Y.;Gu,J.;Hagner-McWhirter,Å.Western Blotting via Proximity Ligation for High Performance Protein Analysis. Molecular \& Cellular Proteomics. 2011,10(11).

68. Jin,S.;Furtaw,M.D.;Chen,H.Multiplexed Western Blotting Using Microchip Electrophoresis. Analytical Chemistry. 2016,88(13),6703-6710.

69. Mishra,M.;Tiwari,S.;Gomes,A.V. Protein purification and analysis: next generation Western blotting techniques. Expert Review of Proteomics. 2017,14(11),1037-1053. 